\title{
Feasibility of a multi-modal exercise program on cognition in older adults with Type 2 diabetes - a pilot randomised controlled trial
}

\author{
M. L. Callisaya ${ }^{1,2,6^{*}}$, R. M. Daly ${ }^{3}$, J. E. Sharman ${ }^{1}$, D. Bruce ${ }^{4}$, T. M. E. Davis ${ }^{4}$, T. Greenaway ${ }^{1}$, M. Nolan ${ }^{1}$, R. Beare ${ }^{2,5}$,
} M. G. Schultz' ${ }^{1}$ T. Phan², L. C. Blizzard ${ }^{1}$ and V. K. Srikanth ${ }^{6,7,1,2}$

\begin{abstract}
Background: Type 2 Diabetes (T2D) is associated with increased risk of dementia. We aimed to determine the feasibility of a randomised controlled trial (RCT) examining the efficacy of exercise on cognition and brain structure in people with T2D.

Methods: A 6-month pilot parallel RCT of a progressive aerobic- and resistance-training program versus a gentle movement control group in people with T2D aged 50-75 years $(n=50)$ at the University of Tasmania, Australia. Assessors were blinded to group allocation. Brain volume (total, white matter, hippocampus), cortical thickness and white matter microstructure (fractional anisotrophy and mean diffusivity) were measured using magnetic resonance imaging, and cognition using a battery of neuropsychological tests. Study design was assessed by any changes (during the pilot or recommended) to the protocol, recruitment by numbers screened and time to enrol 50 participants; randomisation by similarity of characteristics in groups at baseline, adherence by exercise class attendance; safety by number and description of adverse events and retention by numbers withdrawn.

Results: The mean age of participants was 66.2 (SD 4.9) years and 48\% were women. There were no changes to the design during the study. A total of 114 people were screened for eligibility, with 50 participants with T2D enrolled over 8 months. Forty-seven participants (94\%) completed the study (23 of 24 controls; 24 of 26 in the intervention group). Baseline characteristics were reasonably balanced between groups. Exercise class attendance was $79 \%$ for the intervention and $75 \%$ for the control group. There were 6 serious adverse events assessed as not or unlikely to be due to the intervention. Effect sizes for each outcome variable are provided.
\end{abstract}

Conclusion: This study supports the feasibility of a large scale RCT to test the benefits of multi-modal exercise to prevent cognitive decline in people with T2D. Design changes to the future trial are provided.

Trial registration: ANZCTR 12614000222640; Registered 3/3/2014; First participant enrolled 26/6/2014, study screening commenced 1/9/2014; Australian and New Zealand Clinical Trial Registry.

Keywords: Dementia, Cognition, Type 2 diabetes, Exercise, Intervention, MRI brain

\footnotetext{
* Correspondence: michele.callisaya@utas.edu.au

${ }^{1}$ Menzies Institute for Medical Research Tasmania, University of Tasmania, 17

Liverpool Street, Hobart, Tasmania, Australia

${ }^{2}$ Stroke and Aging Research Group, Department of Medicine, Southern

Clinical School, Monash University, Clayton, Victoria, Australia

Full list of author information is available at the end of the article
} 


\section{Background}

Worldwide there are over 35 million people living with dementia, with numbers projected to reach close to 115 million by 2050 [1]. Dementia is the greatest cause of disability and associated with significant decline in quality of life. Dementia was recently highlighted as a major societal issue due to the absence of effective diseasemodifying medications [2]. Strategies for prevention in people at high risk are therefore important in reducing the current and future burden of dementia on the individual and the health-care system.

Type 2 Diabetes (T2D) is regarded as one of the most important modifiable risk factors for dementia [1]. T2D is associated with a nearly two-fold increase in the risk of incident dementia [3] and with accelerated rates of cognitive decline [4]. The predominate cognitive domains affected include executive function and processing speed, and to a lesser degree verbal and visual memory [5]. The pathways leading to cognitive decline in T2D appear to be mixed with both neurodegeneration $[6,7]$ and cerebrovascular disease [7, 8] playing roles. Given the presence of multiple risk factors and pathophysiological mechanisms (dysglycaemia, insulin resistance, obesity, hypertension, physical inactivity) $[9,10]$ contributing to $\mathrm{T} 2 \mathrm{D}$ related dementia, therapeutic approaches that can address multiple risk factors simultaneously are likely to provide the greatest benefits.

Structured exercise training for at least 3 to 6 months has been demonstrated to improve glycaemic, metabolic, inflammatory and vascular profiles in people with T2D [11]. Exercise for animals has also been shown to improve angiogenesis, synaptogenesis and neurogenesis [12]. Although there is persuasive evidence from observational studies regarding the benefits of exercise for cognitive health [13], results from human randomised controlled trials (RCT) have been inconsistent in older people [14-19]. A recent meta-analysis of studies of exercise intervention trials in older adults suggests benefits for cognition, for both aerobic and resistance training [20]. However, a Cochrane review and a recent RCT found no benefit for exercise in cognitively healthy older people $[14,15]$. Those with T2D represent a distinct high-risk group who have a cluster of biological risk factors for cognitive decline. They may therefore respond favourably to risk factor optimization via a multi-modal exercise program, but the relevant data are limited. A small study of people with pre-diabetes or recently diagnosed diabetes $(n=28)$ found that 6 months of aerobic training improved executive function [21]. In an exploratory post-hoc analysis from the Lifestyle interventions and Independence for Elders (LIFE) RCT $(n=415)$, a 24 month exercise intervention resulted in better scores in global cognitive function and delayed recall only in people with T2D [22]. To date, there has been no adequately powered trial that has demonstrated the efficacy of exercise on cognitive function and other measures of brain health in people with T2D.

Prior to embarking on such a large scale trial, we conducted a pilot RCT in people with T2D to: 1) determine the feasibility (design, recruitment, screening, randomisation, adherence, safety and retention) of a 6-month multi-modal exercise training program compared with control to preserve or improve cognition and brain structure, and 2) provide an estimate of the effects of the intervention on outcomes and MRI brain structure.

\section{Methods \\ Study design}

Cognition and Diabetes in Older Tasmanians - A pilot RCT of exercise (CDOT-X) was a 6-month single blind parallel RCT incorporating a supervised and progressive multi-modal exercise program with blinded outcome assessments. Participants with previously diagnosed T2D were randomised to either: 1) a multi-modal aerobic and resistance training (ART) program, or 2) a stretching/ gentle movement control group. Participants were randomised by a central automated allocation procedure (parallel groups stratified within two age strata of 50-64 and 65-75 years) after baseline assessment, based on computer-generated random numbers ensuring allocation concealment. Assessment staff were blinded to randomisation group. Participants were informed that the study was designed to examine the difference between two different types of exercise, but were unaware of the nature of the alternative exercise program. All assessments and exercise classes (the two study groups trained at separate times) occurred at the Menzies Institute for Medical Research, University of Tasmania. Brain MRI scans were performed at the Royal Hobart Hospital, Tasmania, Australia. The trial was registered with the Australian New Zealand Clinical Trials Registry: ACTRN: $12,614,000,222,640$ and was performed according to the CONSORT 2010 extension to randomised pilot and feasibility trials [23]. Ethics approval was from the Human Ethics Committee Tasmania Network (H0013664) and written informed consent was obtained from all participants.

\section{Participants and eligibility criteria}

Recruitment strategies included contacting participants from a previous longitudinal study of brain health in T2D (Cognition and Diabetes in Older Tasmanians (CDOT) [7], advertising at the Diabetes Clinics at the Royal Hobart Hospital, and by using Australian National Diabetes Services Scheme listings, general advertising, and social media . Eligibility was initially determined by a telephone screening interview (for the inclusion and exclusion criteria listed below). This was followed on a separate day by a medical assessment (including medical 
history, medication use and an ECG) by a doctor and an exercise stress test at the Menzies Institute for Medical Research. During the test VO2max was collected using the Bruce protocol and an exercise ECG and echocardiogram were performed. If the doctor had any concerns about the participants results they were referred for a cardiology review. Participants who passed these assessments were asked to return on a separate day for the clinic assessment and MRI scan.

\section{Inclusion criteria}

1) established T2D diagnosed by fasting blood glucose $\geq 7 \mathrm{mmol} / \mathrm{L}$ or $2 \mathrm{~h}$ post-prandial glucose $\geq 11.1 \mathrm{mmol} / \mathrm{L}$ or $\mathrm{HbA}_{1 \mathrm{C}} \geq 6.5 \%$ according to current American Diabetes Association Guidelines [24]; 2) age 50-75 years; 3 ) willingness and ability to participate in a structured exercise program for 6-months, and 4) ability to participate in all cognitive testing.

\section{Exclusion criteria}

1) Severe orthopaedic or respiratory conditions that would preclude participation in an exercise program, or those with absolute contraindications to exercise according to American College of Sports Medicine guidelines [25]; 2) severe cardiovascular disease detected by an exercise stress test as per Australian guidelines [26]; 3) dementia (Telephone Interview for Cognitive Status TICS-Modified score < 27/50); 4) known central nervous system disorders that may have important effects on cognitive function (e.g. intracranial tumour, multiple sclerosis, Parkinson's disease); 5) contraindication to MRI; 6) existing participation in structured exercise greater than the equivalent of $30 \mathrm{~min}$ per week at moderate intensity.

\section{Aerobic and resistance training intervention}

Participants in the intervention group were asked to undertake a 6-month multi-modal exercise program incorporating aerobic and progressive resistance training (PRT) as this has been found to have the strongest benefit on both cognition [18] and T2D markers such as glucose control [27], as well as potentially benefiting brain health through different signaling pathways [28]. It was implemented by accredited exercise physiologists (AEP) or physiotherapists, who supervised small groups (6-8 people) undertaking two one-hours supervised sessions per week on non-consecutive days, supplemented by a further one-hour per week unsupervised session at the participant's home or in the community with prescribed aerobic exercise. Intensity of training relied on the Borg Scale rating of perceived exertion (RPE; range 6 to 20) [25]. Each supervised session consisted of approximately 30 min of moderate- to high-intensity PRT ( 3 sets of 8-12 reps at $14-17$ 'hard to very hard' on the 20 point RPE scale) of the upper and lower extremity exercises using body weight, machine or free weights [29]. An aerobic component of approximately $30 \mathrm{~min}$ consisted of stationary cycling, cross trainer, rower or treadmill walking starting at a low-moderate intensity (RPE 12-13) and progressively building to a moderate-vigorous intensity (RPE 14-16) [26]. The program was individually tailored to each participants ability and progressive intensity was applied with increasing fitness to ensure safety recommendations were met [26]. The 6-month program was divided into six 4 weekly mesocycles and a final 2 week mesocycle of progressing higher intensity. Within the 4 week cycle the final week was at a slightly lower intensity (regeneration phase), before increasing intensity again in the first week of the following mesocycle.

\section{Control intervention}

Participants randomised to the control arm received a 6month light intensity upper and lower limb stretching and gentle movement program, which was also implemented by AEPs and physiotherapists at a distinct time to the intervention group. Participants attended two one-hour supervised sessions per week, and carried out one unsupervised session at home. These activities were designed to provide the same amount of participative involvement and socialization as the intervention group.

Both groups were provided with a participant manual outlining the group specific exercise program as well as motivational tips and safety advice (e.g. foot inspection, measurement of blood glucose before and after exercise).

\section{Feasibility measures \\ Design}

Ability and time required of study staff to co-ordinate the recruitment, screening and clinic tasks; coordination of exercise and control group classes; ability of participants to attend classes; any changes to the study protocol.

\section{Recruitment and screening}

Time and number of people screened to enrol 50 participants.

\section{Randomisation}

Balance of characteristics in each group.

\section{Adherence}

Adherence in both groups assessed by attendance at the supervised sessions. Percentages of classes attended/allocated in each group. Home exercise training was assessed by a participant exercise diary. 


\section{Safety}

The number and description of both serious (any admission to the department of emergency medicine or hospitalization, life threatening event or results in persistent or significant disability/incapacity or death) and other adverse events by group. Serious adverse events (including falls, injuries, hypoglycaemic events and foot ulcers) were adjudicated by a Data Safety Monitor (an independent academic General Practitioner) as not related to the study, probably not related, unlikely but possibly related or probably related to the study.

\section{Retention}

Number of participants that withdrew by group.

\section{Outcome measures}

Measurements were administered at baseline and 6months at the Menzies Institute for Medical Research, apart from MRI scans. Clinics lasted approximately $3 \mathrm{~h}$ and included breakfast. MRI scans occurred on a separate day at the Royal Hobart Hospital which is located across the road from the Menzies Institute for Medical Research and took approximately $1 \mathrm{~h}$. At the 6 month visit participants were asked not to tell assessment staff which group they attended. All data (except MRIs) were collected on teleform questionnaires, which were scanned and transferred to a secure access database.

\section{Brain MRI}

The following acquisition protocols were used in a $3.0 \mathrm{~T}$ GE Signa HDxt scanner: High-resolution 3-dimensional T1 weighted spoiled gradient recalled (SPGR) $\left(\mathrm{TR}=6.732 \mathrm{~ms}, \mathrm{TE}=2.816 \mathrm{~ms}\right.$, flip angle $=12^{\circ}$, FOV $=225 \mathrm{~mm}$, voxel size $1 \times 1 \times 1 \mathrm{~mm}$; fluid attenuated inversion recovery (FLAIR, $\mathrm{TR}=10,000 \mathrm{~ms}, \mathrm{TE}=121 \mathrm{~ms}$, FOV $=220 \mathrm{~mm}$, flip angle $=90^{\circ}$, voxel size $0.4 \times 0.4$ and $4 \mathrm{~mm}$ ); DTI using High Angular Resolution Diffusion Imaging (HARDI) sequence with 64 gradient directions $\left(\mathrm{b}=2000 \mathrm{~s} / \mathrm{mm}^{2}, \mathrm{TR}=7200 \mathrm{~ms}, \mathrm{TE}=105 \mathrm{~ms}\right.$, FOV $=240 \mathrm{~mm}$, voxel size $(2.0 .954 \times 0.952 .4 \times 6.53 \mathrm{~mm} 3)$ and $1 \mathrm{~b}=0 \mathrm{~s} / \mathrm{mm}^{2}$ reference image. MRI pre-processing and analyses were performed in the image analysis lab at Monash University using well-established methods blinded to age, sex and outcome measures. Brain structural outcome measures included total brain, white matter and hippocampal volume and cortical thickness. The Freesurfer 5.3 longitudinal pipeline [30] was used to estimate cortical thickness and brain volume. Hippocampal volumes were estimated using FSL FIRST [31] with the following additions to improve reliability on older participants. The T1 weighted scans were nonlinearly transformed to MNI space using the transformations estimated by the SPM12 unified segmentation procedure. FIRST was applied to the transformed image and the resulting segmentation transformed back to native space for volume estimation. The FSL TBSS [32] preprocessing pipeline was used to compute a white matter skeleton of the whole brain. Mean white matter microstructural measures, fractional anisotropy (FA) and mean diffusivity (MD), were computed for the skeleton. Cerebrovascular white matter hyperintensities were delineated as previously described [33], with the statistical classifier stage replaced by a manual selection. Lesion masks were used to correct Freesurfer errors caused by hypointensities on T1-weighted scans.

\section{Cognitive function}

The following tests were administered: 1) The Victoria Stroop test (interference score C-D) [34]; 2) The Trail Making Test (shifting score B-A); 3) The Digit Symbol Coding Test (DSC) [35]; The digit span subtest of the Wechsler Adult Intelligence Scale - Third Edition (WAIS-III) [35]; Controlled Oral Word Association Test (COWAT) [36]; The three part Hopkins Verbal Learning Test - Revised (HVLT) [36] and the Rey Complex Figure copy and delay [36]. These tests were chosen as they are sensitive to the effects of T2D [5], and mild cognitive changes that affect daily functioning, and have previously been used in intervention studies in T2D to preserve cognition [37]. In post-hoc analysis we used the cognitive measures that showed improvement in the ART group compared to the control group to calculate a global composite score similar to prior studies [38, 39].

\section{Other measures}

The following measures were also assessed: 1) Brachial and central blood pressure using the IEM Mobil-OGraph with 8 automated readings taken seated every 2 min and then averaged; 2) Fasting blood tests: blood glucose, insulin, $\mathrm{HbA}_{1 \mathrm{C}}$ (baseline only) using standard techniques; 3) Physical fitness: $\mathrm{VO}_{2}$ max obtained using the Bruce treadmill protocol measured on a separate day to the other clinic assessments; 4) Knee extension strength $(\mathrm{kg})$ using a spring gauge from the Short Physiological Performance battery [40] and grip strength using a Jamar digital hand dynamometer (kg); 5) Anthropometrics: Height and weight to calculate BMI and waist circumference using standard measures; and 6) Health and lifestyle: A standardised questionnaire was used to collect data on health, medical history and medication use.

\section{Statistical analysis}

A consort diagram was used to summarise screening, recruitment and study retention. Descriptive statistics were used to describe the characteristics of the sample by group (means [SD], percentages and frequencies) as well as exercise adherence and adverse events. Cognitive and brain measure at baseline and follow-up were 
presented as means and $\mathrm{SE}$ and change over time as means and $95 \% \mathrm{CI}$ by group. Random intercept linear mixed models with maximum likelihood estimation adjusted for age, sex and education (and intracranial volume for MRI measures) were performed to determine if there were any differences in the slope between the groups for the change over time. Correlations coefficients were used to examine associations between change in mechanistic variables $\left(\mathrm{VO}_{2 \max }\right.$, knee extension strength, waist circumference, central and brachial blood pressure, and fasting glucose and insulin levels) and change in a cognitive global score. As this is a pilot study we have not provided $P$-values or controlled for Type 1 errors, but have included mean changes with 95\% confidence intervals (CI) for the within group changes and the net between group differences for the change over time to provide an indication of the magnitude and direction of any effect [41].

\section{Results}

Design

Coordination of recruitment, screening, clinic assessments (3-3.5 h per person) and the interventions were performed without any changes to the protocol during the pilot. Offering classes at multiple times worked well with only one participant unable to attend the two classes per week (see CONSORT diagram Fig. 1). The class size of 6-8 participants in the intervention group $(1 \mathrm{~h})$ and $8-10$ participants in the control group $(1 \mathrm{~h})$ was deemed essential to enable adequate supervision to ensure safety and protocol adherence. Trainers noted that an extra 15-20 min for new participant orientation and for completing exercise charts would be useful. Onsite parking was also considered important by participants.

\section{Recruitment, screening and randomisation}

One-hundred and fourteen participants were screened for eligibility of whom 50 (mean [SD] age 66.2 [4.9] years; $48 \%$ women) were randomised and assigned to the intervention or control group over an 8-month period (September 2014 to May 2015). Reasons for exclusion are shown in Fig. 1 and group characteristics are summarized in Table 1.

Retention: Of the 50 participants, 47 (94\%) remained in the study at the 6-month follow-up. Reasons for withdrawal (ART $n=2$; control $n=1$ ) were unexpected travel interstate $(n=1)$, carer for family member $(n=1)$ or inability to contact $(n=1)$. Of the cognitive assessments one person was colour blind and could not complete the Stroop test. Three people had missing components of the HVLT (delay and recognition $[n=1]$; recognition $[n=2])$. Of the 47 participants who returned at 6 months, 1 further participant was unable to complete the MRI scan due to new claustrophobia. Adherence: Exercise class attendance for the ART group was $79 \%$ and for the controls $75 \%$. Seventy five percent of participants adhered to the $60 \mathrm{~min}$ of home exercise.

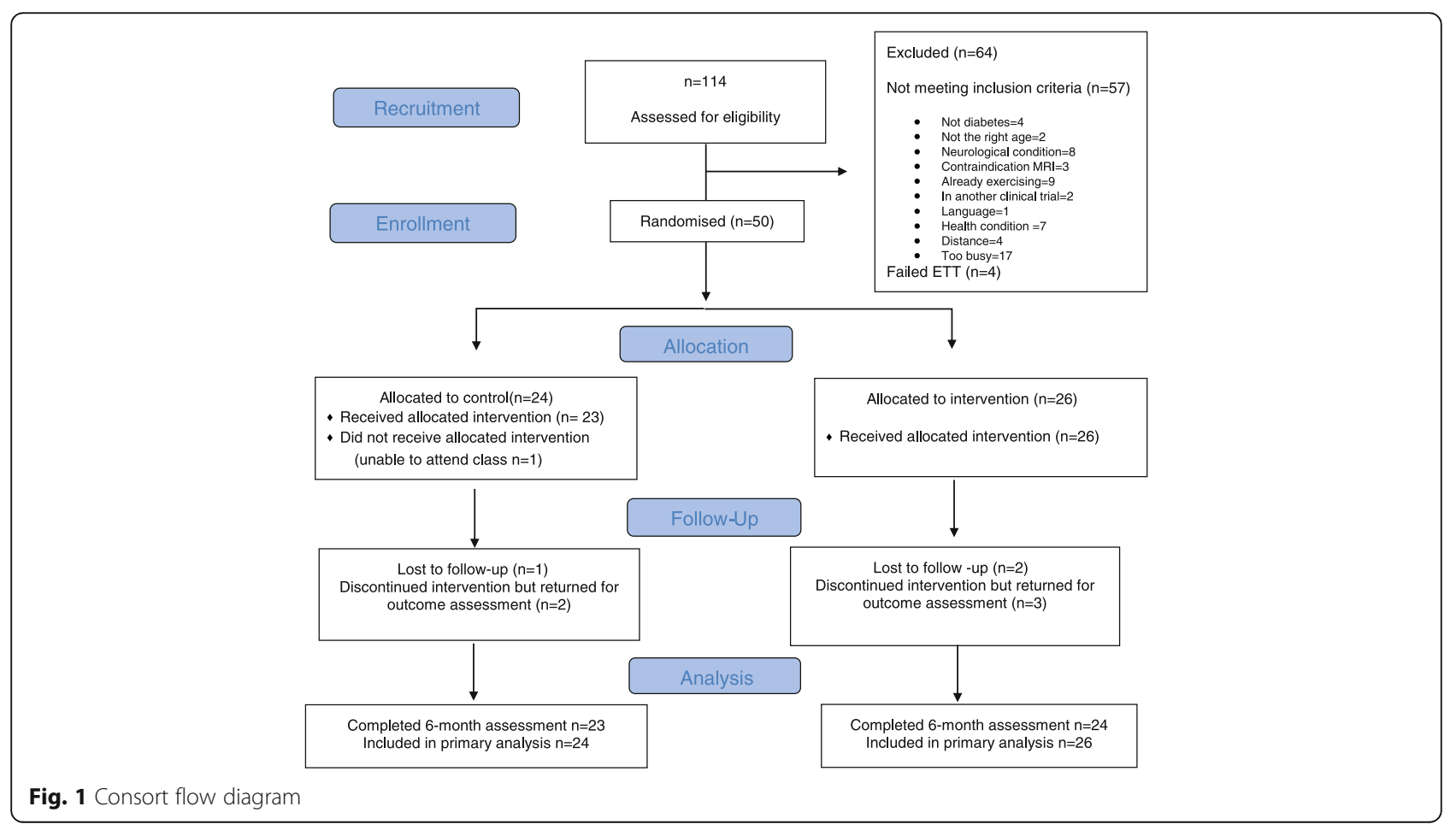


Table 1 Baseline characteristics of the total sample, ART and control group

\begin{tabular}{|c|c|c|c|c|c|c|}
\hline \multirow{2}{*}{$\begin{array}{l}\text { Characteristics, mean, SD unless indicated } \\
\text { Age, years }\end{array}$} & \multicolumn{2}{|c|}{ Total $(n=50)$} & \multicolumn{2}{|c|}{ ART intervention $(n=26)$} & \multicolumn{2}{|c|}{ Control $(n=24)$} \\
\hline & 66.2 & 4.91 & 65.3 & 5.0 & 67.1 & 4.8 \\
\hline Female, n (\%) & 24 & $(48.0)$ & 15 & $(57.7)$ & 9 & $(37.5)$ \\
\hline Body mass index, $\mathrm{kg} / \mathrm{m}^{2}$ & 30.9 & 4.87 & 31.1 & 5.2 & 30.7 & 4.7 \\
\hline Years of education, n (\%) & 12.6 & (3.4) & 13.3 & (3.6) & 11.8 & (3.1) \\
\hline $\mathrm{HbA} 1 \mathrm{c}, \%$ & 6.8 & 1.1 & 6.8 & 1.2 & 6.8 & 1.0 \\
\hline Fasting glucose, mmol/L & 8.3 & 2.6 & 8.5 & 3.0 & 8.1 & 2.1 \\
\hline Fasting insulin, mU/L & 19.1 & 20.6 & 17.8 & 19.5 & 20.6 & 22.2 \\
\hline Systolic blood pressure, mmHg & 117.6 & 12.2 & 117.2 & 10.1 & 118.0 & 14.5 \\
\hline Diastolic blood pressure, mmHg & 77.6 & 10.4 & 77.9 & 8.0 & 77.3 & 12.8 \\
\hline $\mathrm{VO}_{2 \max } \mathrm{L} / \mathrm{min}$ & 21.6 & 4.8 & 22.0 & 5.1 & 21.1 & 4.5 \\
\hline \multicolumn{7}{|l|}{ Self-reported medical history } \\
\hline Hypertension, n (\%) & 44 & $(88.0)$ & 21 & $(80.8)$ & 23 & $(95.8)$ \\
\hline Insulin use, n (\%) & 10 & $(20.0)$ & 6 & $(23.1)$ & 4 & $(16.7)$ \\
\hline High Cholesterol, n (\%) & 42 & $(84.0)$ & 21 & $(80.8)$ & 21 & $(87.5)$ \\
\hline Myocardial infarction, n (\%)* & 2 & $(4.1)$ & 0 & $(0.0)$ & 2 & (8.3) \\
\hline Years since Type 2 Diabetes diagnosis & 12.6 & 7.1 & 11.8 & 7.6 & 13.3 & 6.5 \\
\hline
\end{tabular}

$A R T$ aerobic and resistance training, $H b A 1 c$ glycated hemoglobin, SPPB short physical performance battery

*one participant had missing data

aHypertension: self-reported hypertension or SBPmmHg > 140

\section{Brain MRI}

Table 2 provides the unadjusted and adjusted means for brain MRI measures at baseline and 6 months, the mean change over time in each group, and net between group differences for the change over time (with 95\% CI). Compared with the controls, the ART group showed improvements in white matter integrity (FA), hippocampal and total brain volumes, and less decline in white matter volume over the 6-month period.

\section{Cognition}

Unadjusted and adjusted means for cognitive measures by group at baseline and 6 months, and the mean change and net between group differences (with 95\% CI) are also presented in Table 2. In post-hoc analyses we also created a global cognitive score by forming a composite of these tests calculated as $\mathrm{z}$ scores standardized to the baseline mean and SD [4, 14] (Table 2). Compared with controls, the ART group showed improvements in the global cognitive score, Stroop C-D, Trails A-B, DSC, Hopkins intermediate and recognition scores, COWATword and Rey Complex Copy tests. Table 3 shows the correlations between change in the mechanistic variables and change in the cognitive composite scores by group. The strongest association was seen between an increase in fitness $\left(\mathrm{VO}_{2 \max }\right)$ and increase in global cognition $(r=0.42)$, with a weaker association for reduction in central systolic blood pressure $(r=-0.15)$. An association between global cognition and fasting blood glucose $(r=0.13)$ was in the opposite direction than expected.

\section{Safety: adverse events}

There were two serious adverse events in the intervention group and four in the control (Table 4). All serious adverse events were ruled by the Data Safety Monitor as unlikely or not due to the intervention. One participant suffered a myocardial infarction 1 week after finishing the ART program and this was ruled unlikely but possibly due to the intervention. There were nine other adverse events recorded including two falls in the control group (in the same participant; one fall in the class); one person with symptomatic postural drop during the exercise tolerance test and six (5 ART and 1 control group) with musculoskeletal complaints of which all resolved within a few weeks or specific exercises associated with pain were modified.

\section{Discussion}

The present pilot study has demonstrated feasibility (in study design, recruitment, screening, randomisation, adherence, safety reporting and retention, and effect size estimates) for a subsequent definitive RCT to test whether a multi-modal exercise program incorporating aerobic plus resistance training can provide benefits to cognition and brain structure in people with T2D. Recruitment procedures resulted in 114 people fulfilling the inclusion and exclusion criteria volunteering over 8 months (approximately 14 participants per month). A telephone screening procedure was successful in determining eligibility and availability including identifying those who were too busy, lived too far away or who were 
Table 2 Mean baseline and 6-month scores for cognitive function and brain MRI and the mean within group changes and net between groups differences for the change over time

\begin{tabular}{|c|c|c|c|c|c|c|c|c|c|c|c|c|c|c|}
\hline \multirow{4}{*}{$\begin{array}{l}\text { Outcome } \\
\text { Cognitive global score }\end{array}$} & \multicolumn{6}{|c|}{ ART group } & \multicolumn{6}{|c|}{ Control Group } & \multirow{2}{*}{\multicolumn{2}{|c|}{$\begin{array}{l}\text { Between-group } \\
\text { difference }^{\mathrm{a}}\end{array}$}} \\
\hline & \multirow{2}{*}{\multicolumn{2}{|c|}{$\frac{\text { Baseline }}{\text { Mean (SE) }}$}} & \multirow{2}{*}{\multicolumn{2}{|c|}{$\frac{6 \text {-months }}{\text { Mean (SE) }}$}} & \multirow{2}{*}{\multicolumn{2}{|c|}{$\begin{array}{l}\text { Change } \\
\text { Mean }(95 \% \mathrm{Cl})\end{array}$}} & \multirow{2}{*}{\multicolumn{2}{|c|}{$\frac{\text { Baseline }}{\text { Mean (SE) }}$}} & \multirow{2}{*}{\multicolumn{2}{|c|}{$\frac{6 \text {-months }}{\text { Mean (SE) }}$}} & \multirow{2}{*}{\multicolumn{2}{|c|}{$\begin{array}{l}\text { Change } \\
\text { Mean }(95 \% \mathrm{Cl})\end{array}$}} & & \\
\hline & & & & & & & & & & & & & \multicolumn{2}{|c|}{ Mean $(95 \% \mathrm{Cl})$} \\
\hline & 0.06 & 0.11 & 0.20 & 0.11 & 0.14 & $0.02,0.27$ & -0.06 & 0.12 & -0.08 & 0.12 & -0.01 & $-0.14,0.12$ & 0.16 & $-0.02,0.34$ \\
\hline RCF copy & 27.6 & 0.9 & 27.4 & 0.9 & -0.2 & $-1.8,1.5$ & 26.2 & 0.9 & 25.9 & 0.9 & -0.3 & $-2.03,1.4$ & 0.2 & $-2.1,2.6$ \\
\hline RCF delay & 14.8 & 1.0 & 16.0 & 1.0 & 1.3 & $-0.2,2.8$ & 13.9 & 1.0 & 15.5 & 1.00 & 1.6 & $0.03,3.1$ & -0.3 & $-2.5,1.8$ \\
\hline Stroop C-D & 16.7 & 1.8 & 12.9 & 1.9 & -3.8 & $-7.1,-0.5$ & 20.1 & 1.9 & 18.3 & 1.9 & -1.8 & $-5.1,1.6$ & -2.1 & $-6.7,2.6$ \\
\hline Trails B-A & 40.2 & 4.5 & 31.5 & 4.7 & -8.7 & $-17.9,0.5$ & 38.2 & 4.7 & 33.8 & 4.8 & -4.3 & $-13.8,5.1$ & -4.4 & $-17.7,9.0$ \\
\hline DSC & 58.6 & 2.6 & 60.8 & 2.6 & 2.2 & $0.2,4.2$ & 56.1 & 2.7 & 56.1 & 2.7 & 0.0 & $-2.1,2.1$ & 2.3 & $-0.6,5.2$ \\
\hline Digit span & 19.2 & 0.7 & 18.2 & 0.7 & -1.0 & $-1.9,-0.1$ & 19.9 & 0.7 & 20.6 & 0.7 & 0.7 & $-0.2,1.6$ & -1.7 & $-3.0,-0.5$ \\
\hline Hopkins I & 26.1 & 0.9 & 26.8 & 0.9 & 0.7 & $-0.9,2.2$ & 27.0 & 0.9 & 27.3 & 1.0 & 0.3 & $0 .-1.3,1.9$ & 0.4 & $-1.9,2.7$ \\
\hline Hopkins D & 9.4 & 0.4 & 9.5 & 0.5 & 0.1 & $-0.7,0.9$ & 9.0 & 0.5 & 9.2 & 0.5 & 0.2 & $-0.6,1.0$ & -0.1 & $-1.2,1.0$ \\
\hline Hopkins R & 10.7 & 0.2 & 10.5 & 0.2 & -0.2 & $-0.7,0.4$ & 10.7 & 0.2 & 10.1 & 0.2 & -0.6 & $-1.1,-0.0$ & 0.5 & $-0.3,1.2$ \\
\hline COWAT word & 40.7 & 2.5 & 43.7 & 2.5 & 3.1 & $0.4,5.7$ & 38.3 & 2.6 & 39.7 & 2.6 & 1.4 & $-1.3,4.1$ & 1.7 & $-2.0,5.4$ \\
\hline COWAT Category & 21.0 & 0.9 & 20.1 & 0.9 & -0.9 & $-2.6,0.8$ & 18.7 & 1.0 & 19.3 & 1.0 & 0.6 & $-1.1,2.4$ & -1.5 & $-3.9,1.0$ \\
\hline \multicolumn{15}{|l|}{ MRI brain } \\
\hline Total brain & 1128.5 & 28.6 & 1130.1 & 28.6 & 1.6 & $-2.3,5.5$ & 1176.14 & 28.55 & 1175.7 & 28.6 & -0.5 & $-4.4,3.4$ & 1.9 & $-3.7,7.4$ \\
\hline White matter & 742.2 & 34.7 & 741.1 & 34.7 & -1.1 & $-9.9,7.7$ & 814.46 & 34.66 & 808.7 & 34.7 & -5.8 & $-14.6,3.1$ & 4.3 & $-8.4,17.0$ \\
\hline C-thickness & 3.8 & 0.1 & 3.8 & 0.1 & 0.0 & $-0.1,1.1$ & 3.43 & 0.13 & 3.5 & 0.1 & 0.1 & $-0.0,0.2$ & -0.0 & $-0.2,0.1$ \\
\hline Hippocampal & 6.9 & 0.1 & 7.0 & 0.1 & 0.10 & $-0.01,0.20$ & 7.2 & 0.14 & 7.1 & 0.1 & -0.02 & $-0.12,0.08$ & 0.13 & $-0.03,0.29$ \\
\hline White FA & 0.408 & 0.004 & 0.412 & 0.004 & 0.002 & $-0.002,0.006$ & 0.406 & 0.004 & 0.399 & .005 & -0.007 & $-0.012,-0.003$ & 0.009 & $0.003,0.016$ \\
\hline White MD & 0.001 & 0.000 & 0.001 & 0.000 & -0.000 & $-0.000,0.000$ & 0.001 & 0.000 & 0.001 & 0.000 & -0.000 & $-0.000,0.000$ & -0.000 & $0.000,0.000$ \\
\hline
\end{tabular}

RCF Rey Complex Figure, DSC digit symbol coding, Hopkins (I-immediate, $D$ delay, $R$ recall), COWAT Controlled Oral Word Association Test, $C$-thickness cortical thickness, FA fractional anisotrophy, MD mean diffusion, Stroop and Trails:higher scores poorer performance

adjusted for age, sex and education (brain measures also adjusted for intracranial volume)

unable to participate for other health reasons. The faceto-face medical assessment and exercise stress test were useful in excluding a further seven people due to high cardiovascular risk. The rate of recruitment was also perceived by trainers as optimal in order to allocate sufficient time for orientating new participants to exercises. Taking into account the relatively small sample size, baseline characteristics appeared balanced between groups except for sex and prior myocardial infarction. It is likely that a larger sample size would further improve the comparability of the intervention and control groups.

The intervention group combined aerobic and resistance training, which is based on superior outcomes previously observed with cognition [18] and T2D markers such as blood glucose control [27], as well as potentially benefiting brain health through different signaling pathways [28]. The control group carried out stretches and gentle movements designed to provide the same amount of socialization as the intervention group. The adherence for the two centre-based sessions was similar (79\% in the intervention and 75\% for the control group), which suggests that participants accepted the outcome of randomisation. The retention rate (94\%) was comparable or better than prior studies described in a metaanalysis [42] with only three people not returning for follow-up. We established the need for small exercise groups to ensure the program was tailored according to each individual, with close supervision by a trainer to enhance motivation, ensure correct exercise technique and appropriate blood glucose monitoring. Six serious adverse events (all in different participants) were deemed not or unlikely due to the intervention. Of other adverse events, there was one fall without injury that occurred in

Table 3 Pearson's correlations between change in mechanistic variables and change in global cognitive function in all participants combined

\begin{tabular}{|c|c|c|c|c|c|c|c|c|c|c|}
\hline & $\begin{array}{l}\text { Waist } \\
\text { circumference }\end{array}$ & $\mathrm{VO}_{2 \max }$ & $\begin{array}{l}\text { Brachial } \\
\text { SBP }\end{array}$ & $\begin{array}{l}\text { Brachial } \\
\text { DBP }\end{array}$ & $\begin{array}{l}\text { Central } \\
\text { SBP }\end{array}$ & $\begin{array}{l}\text { Central } \\
\text { DBP }\end{array}$ & $\begin{array}{l}\text { Knee } \\
\text { strength }\end{array}$ & $\begin{array}{l}\text { Fasting } \\
\text { glucose }\end{array}$ & $\begin{array}{l}\text { Fasting } \\
\text { insulin }\end{array}$ & $\begin{array}{l}\text { Grip } \\
\text { strength }\end{array}$ \\
\hline Global cognitive score & 0.08 & 0.45 & -0.08 & -0.10 & -0.15 & -0.05 & 0.03 & 0.13 & 0.01 & 0.11 \\
\hline
\end{tabular}


Table 4 Participants reporting adverse events

\begin{tabular}{llll}
\hline & Total & Intervention & Control \\
\hline Serious adverse events & 6 & 2 & 4 \\
Cardiac problem $^{\text {a }}$ & 2 & 1 & 1 \\
High blood pressure $^{\mathrm{a}}$ & 1 & 0 & 1 \\
Joint surgery $^{\mathrm{a}}$ & 1 & 1 & 0 \\
Gall bladder $^{\mathrm{a}}$ & 1 & 0 & 1 \\
Allergy $^{\mathrm{b}}$ & 1 & 0 & 1 \\
Important adverse events $_{\text {Fall }}$ & 9 & 5 & 4 \\
Musculoskeletal pain & 2 & 0 & $2^{c}$ \\
Symptomatic postural drop during ETT & 1 & 0 & 1 \\
\hline
\end{tabular}

ETT exercise tolerance test

${ }^{a}$ Required hospitalization

${ }^{b}$ required Emergency Department admission

'Both in the same participant

the control group. Five participants also reported musculoskeletal pain in the intervention group and one in the control group, all of which were pre-existing or resolved with expectant management. It is difficult to compare these figures to other trials as a prior report of adverse events in 121 exercise RCT found that in general, adverse events are poorly reported [43]. In order of prevalence, musculoskeletal complaints, falls and cardiovascular events were most common [43]. Older people and those with T2D are more likely to have conditions such as arthritis and cardiovascular disease. In light of this, we took precautions by performing a medical screen and exercise stress test before randomisation, providing participants with a booklet of the training program and exercise precautions, as well as having a 4 week light intensity preparatory training phase at the start of the program, and one easier week in every 4 week cycle. However, a further individualized initial orientation and assessment at the beginning of the program with the trainer may also be worthwhile.

As this was a pilot study, no a priori power estimations were performed, and no tests of efficacy or statistical significance are provided for the between group differences or change over time in brain or cognitive measures as false positives and false negatives are likely. However, 95\% confidence interval were provided to give information about the magnitude and direction of an effect $[23,41]$. Nevertheless, our between-group difference for the change over time in the global cognitive score was similar to the change (mean decrease 0.18 SD) observed over 5 -years in a previous observational study in people with T2D [4]. These data suggest that a global composite score can be a sensitive primary outcome measure in a larger definitive exercise trial, avoiding issues related to multiple primary outcome measures [44]. Of the individual cognitive scores, all but the Rey
Complex Figure delay, Digit span, Hopkins Delay and the COWAT Categories tests favored the intervention group. We included structural brain and cognitive measures that have previously been shown to be sensitive to T2D related cognitive impairment $[5,7,45]$. Few prior RCT have included brain MRI as well as measurements to study underlying pathways influenced by exercise. In the few brain imaging studies of people without T2D, as little as 6-12 months of exercise has been shown to lead to measurable increases volume of the hippocampus $[46,47]$. Our pilot data are broadly consistent with these findings and suggest that total, white matter and hippocampal volume, as well as white matter integrity might benefit from an ART program. This needs to be confirmed in a larger trial.

Although we foresee no major changes to the protocol in a larger study, there are a number of considerations in the design and conduct of future such trials. Firstly, 12 participants did not complete the $\mathrm{VO}_{2 \max }$ test at followup. Although we did not record the reason for this, wearing a mask for this test and exercising to maximum levels may have caused discomfort to some participants. Sub-maximal tests that reliably estimate $\mathrm{VO}_{2 \max }$ may be easier to complete in future trials. Secondly, although there was no indication that assessment staff were aware of group allocation, we did not formally evaluate blinding such as by asking blinded study staff which group that thought participants were in. Thirdly, exercise trainers indicated that heart rate monitoring would assist in assessing intensity of aerobic exercise training over and above the RPE scale. Fourthly, the treatment period was relatively short. The optimum length of the intervention that is required to prevent long-term cognitive decline is unknown. Prior studies have reported benefits of both kinds of exercise (aerobic and resistance) on blood pressure, glycaemic control, insulin sensitivity, and fat mass within 3-6 months [11]. A meta-analysis of exercise for cognitive function in older adults found the largest benefits with 3-12 months following aerobic plus resistance training [18]. However, many of the benefits of exercise may disappear after a program is stopped. Encouragingly, one study reported that a 24-week walking program was efficacious in improving cognition in older people with MCI one-year post intervention [19]. This suggests that a short-term (6-months) program may boost cognitive reserve over the longer term. A larger definitive study based on this pilot is planned to incorporate and evaluate a 12-month phase designed to assist participants incorporate the program into real world home or community exercise programs. Finally it is possible that some medications such as insulin therapy and the number of adverse events may influence results. These should be considered in the statistical plan and power calculations of a future study. 
In addition to the above considerations there are other limitations to the design of our study. The large number of cognitive tests may have led to fatigue and a decline in performance. However the order of tests was similar for all participants. The lack of follow-up over longer periods means that we were unable to determine longer term dropout rates, effects on cognition or conversion to dementia. It is possible that some participants in the control group may have started to exercise, but it is unlikely that they took up training at the intensity performed by the intervention group. They also attended the control classes twice a week which would have left less time to take up another new exercise program. It is possible that the control class may also have provided cognitive benefits, reducing the differences between groups. Although the combination of aerobic and resistance training may provide additional benefit, a four armed study (aerobic, resistance, aerobic and resistance, control) would be required to tease out their individual effects. Finally, some participants were from the longitudinal CDOT study which may limit generalizability. However, these people are well characterized and were also recruited from the Australian National Diabetes Service Scheme.

\section{Conclusion}

In conclusion, the present pilot study demonstrates strong feasibility in terms of design, recruitment, screening, adherence, safety and retention to a multimodal exercise program. A larger definitive trial to determine if exercise can delay or prevent cognitive decline in this high-risk group is now required. A delay in just 1 year of the onset of dementia may result in 9.2 million fewer cases globally [48].

\section{Acknowledgements}

Not applicable

Funding

Alzheimers Australia Research Foundation Grant No. CF13/3559.

\section{Availability of data and materials}

The datasets used and/or analysed during the current study are available from the corresponding author on reasonable request.

\footnotetext{
Authors' contributions

1. MC, RD, JS, DB, TG, MN, RB, MS, TP, LB and VS made substantial contributions to conception and design, or acquisition of data, or analysis and interpretation of data; 2. MC, RD, JS, TD, MN, RB, LB, TP, VS been involved in drafting the manuscript or revising it critically for important intellectual content; 3. MC, RD, JS, DB, TD, TG, MN, RB, MS, TP, LB, VS have given final approval of the version to be published. Each author should have participated sufficiently in the work to take public responsibility for appropriate portions of the content; and 4. MC, LB and VS agreed to be accountable for all aspects of the work in ensuring that questions related to the accuracy or integrity of any part of the work are appropriately investigated and resolved.
}

Ethics approval and consent to participate

Ethics approval was from the Human Ethics Committee Tasmania Network (H0013664) and written informed consent was obtained from all participants.

Consent for publication

Not applicable

\section{Competing interests}

The authors declare that they have no competing interests.

\section{Publisher's Note}

Springer Nature remains neutral with regard to jurisdictional claims in published maps and institutional affiliations.

\section{Author details \\ ${ }^{1}$ Menzies Institute for Medical Research Tasmania, University of Tasmania, 17 Liverpool Street, Hobart, Tasmania, Australia. ${ }^{2}$ Stroke and Aging Research Group, Department of Medicine, Southern Clinical School, Monash University, Clayton, Victoria, Australia. Institute for Physical Activity and Nutrition (IPAN), School of Exercise and Nutrition Sciences, Faculty of Health, Deakin University, Melbourne, Victoria, Australia. ${ }^{4}$ Medical School, Faculty of Health and Medical Sciences, University of Western Australia Fremantle Hospital, Fremantle, Western Australia, Australia. ${ }^{5}$ Murdoch Children's Research Institute, Royal Children's Hospital, Melbourne, Australia. ${ }^{6}$ Peninsula Clinical School, Central Clinical School, Monash University, Melbourne, Victoria, Australia. 'Department of Medicine, Frankston Hospital, Peninsula Health, Melbourne, Victoria, Australia.}

Received: 13 July 2017 Accepted: 8 October 2017

Published online: 16 October 2017

\section{References}

1. Alzheimer's Disease International: World Alzheimer report 2014. London. Alzheimers Disease International; 2014. https://www.alz.co.uk/research/ WorldAlzheimerReport2014.pdf.

2. G8 Health and Science Ministers. G8 dementia summit declaration. In: Edited by Gov UK, editor. RDD/10495; 2013.

3. Cheng G, Huang C, Deng H, Wang H. Diabetes as a risk factor for dementia and mild cognitive impairment: a meta-analysis of longitudinal studies. Intern Med J. 2012;42(5):484-91.

4. Nooyens AC, Baan CA, Spijkerman AM, Verschuren WM. Type 2 diabetes and cognitive decline in middle-aged men and women: the Doetinchem cohort study. Diabetes Care. 2010;33(9):1964-9.

5. Palta P, Schneider AL, Biessels GJ, Touradji P, Hill-Briggs F. Magnitude of cognitive dysfunction in adults with type 2 diabetes: a meta-analysis of six cognitive domains and the most frequently reported neuropsychological tests within domains. J Int Neuropsychol Soc. 2014;20(3):278-91.

6. Moran C, Beare R, Phan TG, Bruce DG, Callisaya ML, Srikanth V. Alzheimer's disease neuroimaging I: Type 2 diabetes mellitus and biomarkers of neurodegeneration. Neurology. 2015;85(13):1123-30.

7. Moran C, Phan TG, Chen J, Blizzard L, Beare R, Venn A, Munch G, Wood AG, Forbes J, Greenaway TM, et al. Brain atrophy in Type 2 diabetes: regional distribution and influence on cognition. Diabetes Care. 2013;36(12):4036-42.

8. Qiu C, Sigurdsson S, Zhang Q, Jonsdottir MK, Kjartansson O, Eiriksdottir G, Garcia ME, Harris TB, van Buchem MA, Gudnason V, et al. Diabetes, markers of brain pathology and cognitive function: the age, gene/environment susceptibility-Reykjavik study. Ann Neurol. 2014;75(1):138-46.

9. Biessels GJ, Strachan MW, Visseren FL, Kappelle LJ, Whitmer RA. Dementia and cognitive decline in type 2 diabetes and prediabetic stages: towards targeted interventions. Lancet Diabetes Endocrinol. 2014;2(3):246-55.

10. Callisaya ML, Moran C, Srikanth VK. Type 2 diabetes mellitus as a causal factor for dementia - is there sufficient evidence from interventional studies? Australian Epidemiologist. 2013;20:1

11. Thomas DE, Elliott EJ, Naughton GA. Exercise for type 2 diabetes mellitus. Cochrane Database Syst Rev. 2006;3:CD002968.

12. van Praag H, Shubert T, Zhao C, Gage FH. Exercise enhances learning and hippocampal neurogenesis in aged mice. J Neurosci. 2005;25(38):8680-5.

13. Brown BM, Peiffer JJ, Martins RN. Multiple effects of physical activity on molecular and cognitive signs of brain aging: can exercise slow 
neurodegeneration and delay Alzheimer's disease? Mol Psychiatry. 2013;18(8):864-74

14. Sink KM, Espeland MA, Castro CM, Church T, Cohen R, Dodson JA, Guralnik J, Hendrie HC, Jennings J, Katula J, et al. Effect of a 24-month physical activity intervention vs health education on cognitive outcomes in sedentary older adults: the LIFE randomized trial. JAMA. 2015;314(8):781-90.

15. Young J, Angevaren M, Rusted J, Tabet N. Aerobic exercise to improve cognitive function in older people without known cognitive impairment. Cochrane Database Syst Rev. 2015;4:CD005381.

16. Fiatarone Singh MA, Gates N, Saigal N, Wilson GC, Meiklejohn J, Brodaty H, Wen W, Singh N, Baune BT, Suo C, et al. The Study Of Mental and Resistance Training (SMART) study-resistance training and/or cognitive training in mild cognitive impairment: a randomized, double-blind, doublesham controlled trial. J Am Med Dir Assoc. 2014;15(12):873-80.

17. Gates N, Fiatarone Singh MA, Sachdev PS, Valenzuela M. The effect of exercise training on cognitive function in older adults with mild cognitive impairment: a meta-analysis of randomized controlled trials. Am J Geriatr Psychiatry. 2013;21(11):1086-97.

18. Colcombe S, Kramer AF. Fitness effects on the cognitive function of older adults: a meta-analytic study. Psychol Sci. 2003;14(2):125-30.

19. Lautenschlager NT, Cox KL, Flicker L, Foster JK, van Bockxmeer FM, Xiao J, Greenop KR, Almeida OP. Effect of physical activity on cognitive function in older adults at risk for Alzheimer disease: a randomized trial. JAMA. 2008;300(9):1027-37.

20. Northey JM, Cherbuin N, Pumpa KL, Smee DJ, Rattray B. Exercise interventions for cognitive function in adults older than 50: a systematic review with meta-analysis. Br J Sports Med. 2017; doi: 10.1136/bjsports-2016-096587.

21. Baker LD, Frank LL, Foster-Schubert K, Green PS, Wilkinson CW, McTiernan A, Cholerton BA, Plymate SR, Fishel MA, Watson GS, et al. Aerobic exercise improves cognition for older adults with glucose intolerance, a risk factor for Alzheimer's disease. J Alzheimers Dis. 2010;22(2):569-79.

22. Espeland MA, Lipska K, Miller ME, Rushing J, Cohen RA, Verghese J, McDermott MM, King AC, Strotmeyer ES, Blair SN, et al. Effects of physical activity intervention on physical and cognitive function in sedentary adults with and without diabetes. J Gerontol A Biol Sci Med Sci. 2017;72(6):861-6.

23. Eldridge SM, Chan CL, Campbell MJ, Bond CM, Hopewell S, Thabane L, Lancaster GA. group Pc: CONSORT 2010 statement: extension to randomised pilot and feasibility trials. BMJ. 2016;355:15239.

24. American Diabetes A. (2) Classification and diagnosis of diabetes. Diabetes Care. 2015;38 Suppl:S8-S16.

25. Medicine ACoS: ACSMs guidelines of exercise testing and prescription, 9 edn. Philadelphia: Lippincott Williams \& Wilkins; 2014.

26. Hordern MD, Dunstan DW, Prins JB, Baker MK, Singh MA, Coombes JS. Exercise prescription for patients with type 2 diabetes and pre-diabetes: a position statement from exercise and sport science Australia. J Sci Med Sport. 2012;15(1):25-31.

27. Church TS, Blair SN, Cocreham S, Johannsen N, Johnson W, Kramer K, Mikus CR, Myers V, Nauta M, Rodarte RQ, et al. Effects of aerobic and resistance training on hemoglobin A1c levels in patients with type 2 diabetes: a randomized controlled trial. JAMA. 2010;304(20):2253-62.

28. Cassilhas RC, Lee KS, Fernandes J, Oliveira MG, Tufik S, Meeusen R, de Mello MT. Spatial memory is improved by aerobic and resistance exercise through divergent molecular mechanisms. Neuroscience. 2012;202:309-17.

29. Gianoudis J, Bailey CA, Ebeling PR, Nowson CA, Sanders KM, Hill K, Daly RM Effects of a targeted multimodal exercise program incorporating high-speed power training on falls and fracture risk factors in older adults: a communitybased randomized controlled trial. J Bone Miner Res. 2014;29(1):182-91.

30. Reuter M, Rosas HD, Fischl B. Highly accurate inverse consistent registration: a robust approach. Neurolmage. 2010;53(4):1181-96.

31. Patenaude B, Smith SM, Kennedy DN, Jenkinson M. A Bayesian model of shape and appearance for subcortical brain segmentation. Neurolmage. 2011:56(3):907-22.

32. Smith SM, Jenkinson M, Johansen-Berg H, Rueckert D, Nichols TE, Mackay CE, Watkins KE, Ciccarelli O, Cader MZ, Matthews PM, et al. Tract-based spatial statistics: voxelwise analysis of multi-subject diffusion data. Neurolmage. 2006;31(4):1487-505.

33. Beare R, Srikanth V, Chen J, Phan TG, Stapleton J, Lipshut R, Reutens D. Development and validation of morphological segmentation of age-related cerebral white matter hyperintensities. Neurolmage. 2009;47(1):199-203.
34. Spreen O, Strauss E. A compendium of neuropsychological tests. Administration, norms, and commentary. 2nd ed. New York: Oxford University Press; 1998.

35. Weschler D. Weschler Adult Intelligence Scale. New York: Psychological Corporation; 1997.

36. Lezak M. Neuropsychological assessment. 3rd ed. New York: Oxford University Press; 1995

37. Williamson JD, Launer LJ, Bryan RN, Coker LH, Lazar RM, Gerstein HC, Murray AM, Sullivan MD, Horowitz KR, Ding J, et al. Cognitive function and brain structure in persons with type 2 diabetes mellitus after intensive lowering of blood pressure and lipid levels: a randomized clinical trial. JAMA Intern Med. 2014;174(3):324-33.

38. Valls-Pedret C, Sala-Vila A, Serra-Mir M, Corella D, de la Torre R, MartinezGonzalez MA, Martinez-Lapiscina EH, Fito M, Perez-Heras A, Salas-Salvado J, et al. Mediterranean diet and age-related cognitive decline: a randomized clinical trial. JAMA Intern Med. 2015;175(7):1094-103.

39. Barnes DE, Santos-Modesitt W, Poelke G, Kramer AF, Castro C, Middleton LE, Yaffe K. The mental activity and eXercise (MAX) trial: a randomized controlled trial to enhance cognitive function in older adults. JAMA Intern Med. 2013;173(9):797-804.

40. Lord SR, Menz HB, Tiedemann A. A physiological profile approach to falls risk assessment and prevention. Phys Ther. 2003;83(3):237-52.

41. Leon AC, Davis LL, Kraemer HC. The role and interpretation of pilot studies in clinical research. J Psychiatr Res. 2011;45(5):626-9.

42. Smith PJ, Blumenthal JA, Hoffman BM, Cooper H, Strauman TA, Welsh-Bohmer $K$, Browndyke JN, Sherwood A. Aerobic exercise and neurocognitive performance: a meta-analytic review of randomized controlled trials. Psychosom Med. 2010;72(3):239-52.

43. Liu CJ, Latham N. Adverse events reported in progressive resistance strength training trials in older adults: 2 sides of a coin. Arch Phys Med Rehabil. 2010;91(9):1471-3.

44. Langbaum JB, Hendrix S, Ayutyanont N, Bennett DA, Shah RC, Barnes LL, Lopera F, Reiman EM, Tariot PN. Establishing composite cognitive endpoints for use in preclinical Alzheimer's disease trials. J Prev Alzheimers Dis. 2015;2(1):2-3.

45. Reijmer YD, Leemans A, Brundel M, Kappelle L, Biessels GJ. Utrecht vascular cognitive impairment study G: disruption of the cerebral white matter network is related to slowing of information processing speed in patients with type 2 diabetes. Diabetes. 2013;62(6):2112-5.

46. Colcombe SJ, Erickson KI, Scalf PE, Kim JS, Prakash R, McAuley E, Elavsky S, Marquez DX, Hu L, Kramer AF. Aerobic exercise training increases brain volume in aging humans. J Gerontol A Biol Sci Med Sci. 2006;61(11):1166-70.

47. Erickson KI, Voss MW, Prakash RS, Basak C, Szabo A, Chaddock L, Kim JS, Heo $\mathrm{S}$, Alves $\mathrm{H}$, White $\mathrm{SM}$, et al. Exercise training increases size of hippocampus and improves memory. Proc Natl Acad Sci U S A. 2011;108(7):3017-22.

48. Brookmeyer R, Johnson E, Ziegler-Graham K, Arrighi HM. Forecasting the global burden of Alzheimer's disease. Alzheimers Dement. 2007;3(3):186-91.

\section{Submit your next manuscript to BioMed Central and we will help you at every step:}

- We accept pre-submission inquiries

- Our selector tool helps you to find the most relevant journal

- We provide round the clock customer support

- Convenient online submission

- Thorough peer review

- Inclusion in PubMed and all major indexing services

- Maximum visibility for your research

Submit your manuscript at www.biomedcentral.com/submit 